\title{
Atomic-Scale Study of Intrinsic Defects Suppressing the Thermal Conductivity of Boron Arsenide
}

Qiang Zheng $^{{ }^{*}}$, Carlos A. Polanco ${ }^{1}$, Mao-Hua Du ${ }^{1}$, Lucas R. Lindsay ${ }^{1}$, Miaofang Chi $^{2}$, Jiaqiang Yan $^{1,3}$ and Brian C. Sales ${ }^{1}$

1. Materials Science and Technology Division, Oak Ridge National Laboratory, Oak Ridge, TN 37831, USA

2. Center for Nanophase Materials Sciences, Oak Ridge National Laboratory, Oak Ridge, TN 37831, USA

3. Department of Materials Science and Engineering, University of Tennessee, Knoxville, TN 37996, USA

* Corresponding author: zhengq@ornl.gov

As microelectronic devices become faster and smaller, materials with ultrahigh thermal conductivity $(\kappa)$ are becoming important for new generation electronic devices. Recently, based on first-principles calculations, boron arsenide (BAs) with a zinc blende-type cubic structure was predicted to possess an unusually high $\kappa$ at room temperature of over $2000 \mathrm{Wm}^{-1} \mathrm{~K}^{-1}$, comparable to that of diamond [1]. However, the experimentally measured $\kappa$ of BAs single crystals was only $200 \sim 350 \mathrm{Wm}^{-1} \mathrm{~K}^{-1}$ for a few years, an order of magnitude lower than the predicted value [2-3]. Although theoretical calculations [4] and x-ray photoelectron spectroscopy (XPS) studies [2,3] reveal that As vacancies $\left(V_{\mathrm{As}}\right)$, even with very low concentration, could significantly suppress $\kappa, V_{\mathrm{As}}$ in BAs materials has never been directly observed to date. Therefore, direct observation and identification of the intrinsic defects in BAs that suppress $\kappa$ become quite urgent for growth of high-quality BAs crystals or films.

Scanning transmission electron microscopy (STEM) imaging was performed to investigate the intrinsic defects in BAs single crystals at atomic scale. As shown in Figure 1a, the perfect structure of BAs in the projection of [001] reveals each atomic column involving a single type of atoms, either B or As. Two regions were selected for high-angle annular dark-field (HAADF) imaging. Their thicknesses were measured from the corresponding electron energy loss (EEL) spectra in Figure 1b, using the log-ratio method. Figure 1c displays a HAADF image for a region with a thickness of $\sim 4.7 \mathrm{~nm}$. Obvious intensities for some B columns and intensity weakening for some As columns were observed. Such feature was highlighted by the intensity profile in Figure 1d, suggesting the appearance of $\mathrm{As}_{\mathrm{B}}$ antisites and $\mathrm{B}_{\mathrm{As}}$ antisites (or $V_{\mathrm{As}}$ ). Further HAADF image simulation confirms the defect-induced intensity variation.

Figure 1e shows a HAADF image for a much thinner region $(\sim 1.7 \mathrm{~nm}$ thick). As revealed by the intensity profile in Figure 1f, the intensity fluctuation in this HAADF image is strengthened due to its thinner thickness, compared to that in Figure 1c. More HAADF image simulation using the model with antisite pairs is in good agreement with the experimental observations.

Additional calculations on formation energies confirm that the antisite pair is energetically preferred among the different types of point defects. The concentration of such antisite pairs was estimated to be $1.8(8) \%$ from many HAADF images. This can suppress $\kappa$ of BAs single crystal to be $65 \sim 100 \mathrm{Wm}^{-1} \mathrm{~K}^{-1}$ from first principles calculation. This calculated $\kappa$ is in reasonable agreement with the measured value. The schematics for the local antisite pairs scattering phonons is exhibited in Figure 1g. 
Paralleling to this study [5], quite recent work reported the growth of BAs single crystals with thermal conductivity values near $1000 \mathrm{Wm}^{-1} \mathrm{~K}^{-1}$ [6-8]. Based on our work, these new crystals presumably have much less intrinsic antisite-pair defects as compared to previous work. Our work for the first time directly confirmed the dominant type of intrinsic defects in BAs materials at atomic scale. The identification of intrinsic defects in BAs would provide critical information for future potential application of BAs in electronic devices [9].

\section{References:}

[1] L Lindsay et al., Phys. Rev. Lett. 111 (2013), p. 025901.

[2] J Kim et al., App. Phys. Lett. 108 (2016), p. 201905.

[3] B Lv et al., App. Phys. Lett. 106 (2015), p. 074105.

[4] NH Protik et al., Phys. Rev. B 94 (2016), p. 045207.

[5] Q Zheng et al., Phys. Rev. Lett. 121 (2018), p. 105901.

[6] JS Kang et al., Science 361 (2018), p. 575.

[7] S Li et al., Science 361 (2018), p. 579.

[8] F Tian et al., Science 361 (2018), p. 582.

[9] Research supported by the U.S. Department of Energy (DOE), Office of Science, Basic Energy Sciences, Materials Science and Engineering Division and ORNL's CNMS, which is a DOE Office of Science User Facility.
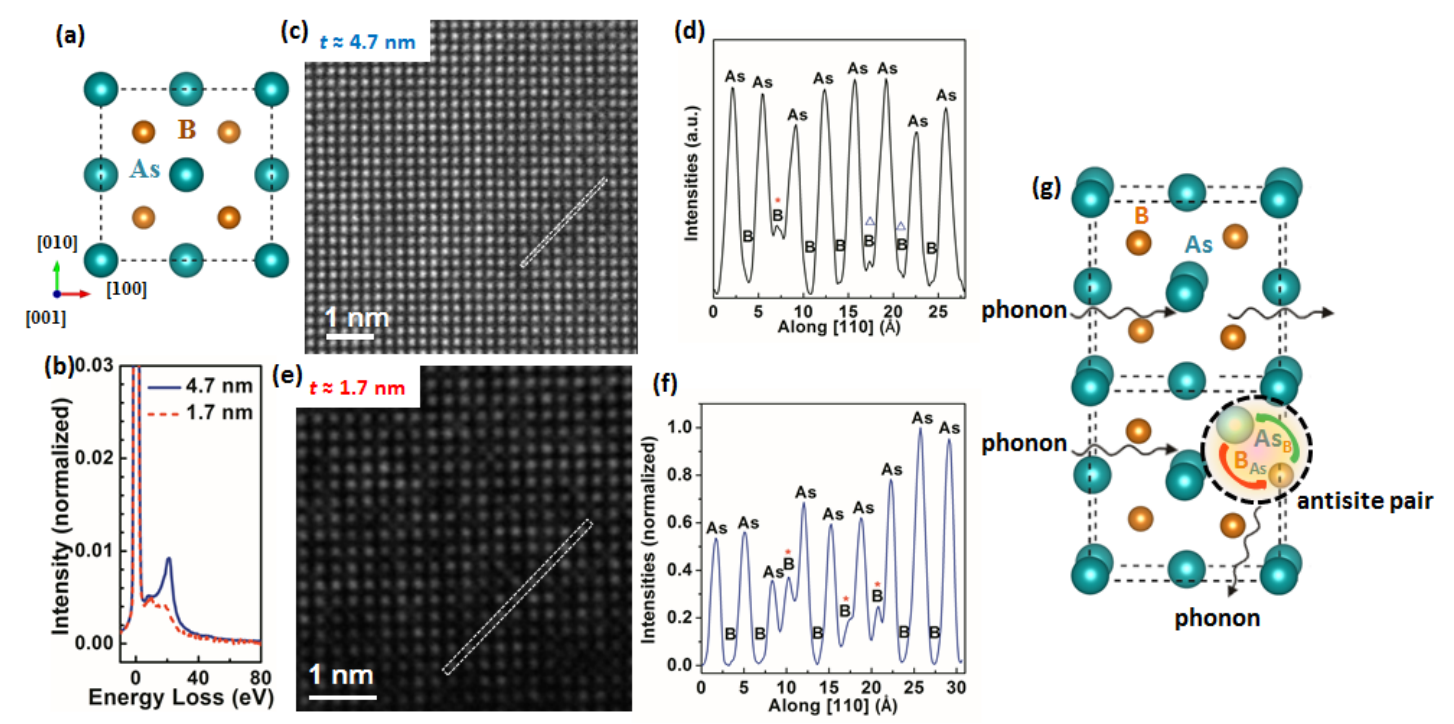

Figure 1. (a) The crystal structure of BAs in the projection of [001]. (b) EEL spectra used to estimate thicknesses for the two HAADF imaging regions. (c) A HAADF image along [001] for a region with a thickness of $4.7 \mathrm{~nm}$. (d) The intensity profile for the dashed rectangular region in (c) reveals As${ }_{B}$ antisite defects and intensity weakening for their neighboring As columns; the two B columns marked by blue triangles indicate likely one $\mathrm{As}_{\mathrm{B}}$ in each while the column marked by the red asterisk reveals more than one $\mathrm{As}_{\mathrm{B}}$ in it. (e) A HAADF image along [001] for a region with a thickness of $1.7 \mathrm{~nm}$. (f) The intensity profile for the dashed rectangular region in (e) indicates more obvious intensity fluctuation due to antisite defects; three B columns marked by red asterisks indicates $\mathrm{As}_{\mathrm{B}}$ in them. (g) A schematics shows local antisite pairs scatter phonons. 